\title{
"Ich rede jetzt nicht direkt persönlich, denn ich bin darüber momentan hinaus ..." \\ Der Suizid als Selbsttechnik der Subjektspaltung am Beispiel von Horváths Glaube, Liebe, Hoffnung
}

„Ich rede jetzt nicht direkt persönlich, denn ich bin darüber momentan hinaus ..."

The Suicide as a Self-Technique of the Subject Fission on the Example of Horváth's Glaube, Liebe, Hoffnung

Erkan Osmanović

\begin{abstract}
Luhmann states that the individuality of people varies from one individuality of inclusion to another exclusive individuality. In stratified societies it was measured by which layer or class one belonged. In functionally differentiated societies there is a fragmentation of the subject. To deal with this experience, Elisabeth in Horváths play Glaube, Liebe, Hoffnung, uses her suicide in the service of a subject fission. The term "subject fission" subsumes various self-techniques that have been used since antiquity and arose in the context of a concern for themselves. To what extent suicide also assumes this function for Elisabeth, is elaborated in the text analysis.
\end{abstract}

Keywords

Horváth, subject fission, Glaube, Liebe, Hoffnung, individuality 


\section{1.}

Im Drama Glaube, Liebe, Hoffnung ${ }^{1}$ zeichnet der österreichische Autor Ödön von Horváth den Werdegang eines weiblichen Subjekts, Elisabeth, nach, deren Selbstkonstituierung innerhalb der reduzierten Möglichkeiten einer Indivualitätserfahrung ${ }^{2}$ scheitert und die schließlich als alleinigen Ausweg aus ihrer misslichen Lage den Suizid wählt.

Elisabeth wird in jeder Lebenssituation auf sich selbst zurückgeworfen, eine soziale Interaktion ist ihr in wiederholten Fällen nicht mehr möglich, sobald einerseits ihre kriminelle Vergangenheit, aber auch ihre Arbeitslosigkeit erkannt wird. In einer arbeitsteiligen Gesellschaft, die Ordnungssysteme wie etwa religiösen Glauben oder romantische Liebe als nicht qualifizierte Bezugssysteme einer Konstituierung des Subjekts ablehnen, wird die Existenzsicherung eines jeden Menschen zwar geduldet - wenn auch stark sanktioniert -, einem arbeitslosen Individuum aber jeglicher Ich-Entwurf verunmöglicht. Denn Elisabeths kriminelle Handlungen sind nicht die Ursache der gesellschaftlichen Ablehnung, sondern Ergebnis einer vorhergehenden Zurückweisung aufgrund des Makels Arbeitslosigkeit, der es ihr nicht ermöglicht sich selbst als handelndes Subjekt zu erleben.

Die Subjektproblematik des modernen Individuums hat Luhmann 1980 in seiner Untersuchung Gesellschaftsstruktur und Semantik aufgezeigt. Er sieht das Problem in der Definition des Ichs durch das Individuum in der Wandlung der gesellschaftlichen Systeme. ${ }^{3}$ Für Luhmann ist das offensichtlichste Merkmal der Wendung hin zur Moderne in der neuen Art und Weise der Differenzierung der Gesellschaft zu finden. Denn in älteren Gesellschaftssystemen, die nach stratifikatorischen Kategorien differenzierten, gab es für die Menschen bestimmte Stände, Kasten oder Schichten, denen sie sich zugehörig fühlten. Aus dieser jeweiligen Zugehörigkeit leiteten die Menschen ihre Rechte und Pflichten ab und waren damit aufgehoben in einem Kontext von Leistung und Gegenleistung, der ihnen die Integration in das jeweilige gesellschaftliche Teilsystem möglich machte. Familien oder Stammhäuser dienten als Instanzen, die dafür sorgten, dass jeder Mensch in nur einem gesellschaftlichen Teilsystem, der jeweiligen Schicht oder dem Stand, eingebettet war. In diesen stratifikatorischen Gesellschaftssystemen wurden die gesellschaftlichen Verhältnisse als naturgegeben angesehen und Kritik an ihnen mit moralischen Verurteilungen entkräftet. Ein weiteres Mittel um diesen Zustand aufrecht zu erhalten, war laut Luhmann die Vorstellung von einer diesseitigen und jenseitigen Existenz, wie sie etwa der religiöse Glaube offeriert. Durch all diese Mittel wurde eine stabile Ordnung der Gesellschaft erreicht, aber auch die „Beschränkung des

1 Der Artikel bezieht sich in seinen Ausführungen auf folgende Ausgabe: HORVÁTH, Ödön von (2007): Glaube, Liebe, Hoffnung. Ein kleiner Totentanz. Frankfurt am Main. Die zitierten Seitenangaben aus dieser Quelle werden im gesamten Artikel mit Klammern im Fließtext markiert.

$2 \mathrm{Zu}$ weiteren literarischen Ausführungen zu Identitätskonstruktionen und -erfahrungen; vgl. BLOCHER, Friedrich K. (1984): Identitätserfahrung. Literarische Beiträge von Goethe bis zu Walser. Köln.

3 Zum folgenden vgl. LUHMANN, Niklas (1998): Individuum, Individualität, Individualismus. In: Luhmann, Niklas: Gesellschaftsstruktur und Semantik. Studien zur Wissenssoziologie der modernen Gesellschaft. Bd. 3. (2. Aufl.). Frankfurt am Main, S. 149-258. 
Zugangs zu Statuspositionen “4 fixiert und in Kauf genommen. Die Basis dieses Systems lag im Einschluss des Individuums in einem festgelegten Teilsystem der stratifikatorischen Gesellschaft. Im Rahmen dieser Einbindung konnte jeder Mensch der älteren Gesellschaften somit eine beschränkte Art der Individualität erreichen, die Luhmann als „Inklusionsindividualität ${ }^{\text {" }}$ bezeichnet. Damit ist eine Art von Individualität gemeint, die sich als die vollkommene Einordnung des Menschen in seinem gesellschaftlichen Teilsystem versteht. Diese Ordnung und das Selbstverständnis der Menschen als Mitglieder einer Gesellschaft änderte sich jedoch im Wandel der stratifikatorischen Gesellschaft hin zum modernen, funktional differenzierten Gesellschaftssystem. In diesen neuen Gesellschaftssystemen erscheint es nicht mehr als normal und möglich für eine Einzelperson sich in nur einem Teilsystem aufzuhalten, sondern jeder Mensch erhält die Möglichkeit sich in verschiedenen gesellschaftlichen Teilsystemen zu betätigen. Denn wo früher die Standeszugehörigkeit den Zugang zu bestimmten Teilsystemen (beispielsweise Recht und Bildung) regelte, wird nun theoretisch jeder Einzelperson der Zutritt durch verschiedene Eingänge ermöglicht. So determiniert zum Beispiel die Teilhabe am Teilsystem der Arbeit nicht mehr diejenige am Teilsystem der politischen Partizipation. Dadurch entsteht die Art von Individualität, die Luhmann als „Exklusionsindividualität ${ }^{“ 6}$ bezeichnet. Der Einzelne sieht seine Individualität als etwas Einzigartiges an, das erst in die Gesellschaft eingeordnet werden muss. Dadurch entsteht ein Bewusstsein für Wahlfreiheit der Lebenskonzepte, die soziale Mobilität wird gesteigert und damit einhergehend die ersten Vorstellungen von allgemeingültigen Menschenrechten.

Trotz all dieser Errungenschaften stößt der Mensch in dieser funktional differenzierten Gesellschaftsordnung auf ein Problem: Durch die Tatsache, dass die unterschiedlichen Teilsysteme die Gesellschaft als Ganzes bilden, ist es für die Gesellschaft nicht möglich sich selbst als Gesamtheit wahrzunehmen. Daraus ergibt sich für das moderne Individuum die Beschwernis, dass es sich an keiner Stelle des Systems mehr als "gesellschaftliches Wesen“7 erleben kann. Daraus ergibt für Luhmann die Problematik des modernen Individuums und damit auch in den Figuren in Glaube, Liebe, Hoffnung abzulesen ist:

„Er [Der/Die Einzelne] kann nur außerhalb der Gesellschaft leben, nur als System eigener Art in der Umwelt der Gesellschaft sich reproduzieren, wobei für ihn die Gesellschaft eine dazu notwendige Umwelt ist. Das Individuum kann nicht mehr durch Inklusion, sondern nur noch durch Exklusion definiert werden. Das ist der strukturelle Grund für die neuartige (post-naturrechtliche) Dramatik von »Individuum und Gesellschaft «. “8

Das Individuum gerät in einen Zwiespalt. Denn der Mensch kann sich nun in verschiedenen funktionalen Rollen erleben (beispielsweise Kunde, Freund, Arbeiter etc.), jedoch

4 Ebd., S. 156.

5 Ebd., S. 160.

6 Ebd.

7 Ebd., S. 158.

8 Ebd. 
wird er dadurch auch in seine Einzelteile zersplittert. Horváths Drama kann als literarisches Exempel gelesen werden, das dieses philosophische Problem aufdeckt. Elisabeth verkörpert das Streben nach Individualität bei gleichzeitigem Wunsch nach Aufhebung und Entgrenzung derselben, und durchlebt damit auf exemplarische Weise die Defizite der arbeitsteiligen Gesellschaft, die erst seit rund 250 Jahren als Gesellschaftssystem in der westlich-geprägten Welt vorherrscht.

Um mit dieser Erfahrung umzugehen, stellt Elisabeth ihren Suizid in den Dienst einer Subjektspaltung. Mit dem Begriff Subjektspaltung werden verschiedene Selbsttechniken subsumiert, die bereits seit der Antike eingesetzt wurden und im Rahmen einer Sorge um sich selbst aufkamen, um sich selbst als Individuum beobachten, also auch als Subjekt verstehen zu können - man denke etwa an den Akt des Lesens, der den Lesenden aufspaltet in Sprecher und Hörer. Pierre Hadot und Michel Foucault konnten das Aufkommen und die Verbreitung dieser Selbsttechniken nachzeichnen und es finden sich viele prominente Namen unter deren Anwendern. ${ }^{9}$ So verweist Platon bereits in seinen Dialogen auf Sokrates und dessen daimon, der als ständiger Begleiter fungiert - bis hinein ins Totenreich. Selbsttechniken dienen der Kreation eines Selbst, wie Thomas Macho 2017 in seinem Band Das Leben nehmen. Suizid in der Moderne erklärt:

„Selbsttechniken ermöglichen und vertiefen eine Aufspaltung des Subjekts: Der Lesende spaltet sich auf in ein sprechendes und ein hörendes Selbst; der Schreibende spaltet sich auf in Autor und Adressat seiner Texte, gleichgültig ob er Dialoge mit sich selbst, Tagebucheintragungen oder Briefe verfasst. “10

Macho verweist auf die Selbsttechnik der meléte thanátou, also die Todesmeditation, die in der europäischen Philosophiegeschichte eine lange Traditionslinie hat und bei deren Ausübung „es darauf ankam, sich in ein transzendentales Selbst - einen Begleiter, Zeugen, Beobachter - und ein empirisches Ich aufzuspalten." ${ }^{11}$ Die Todesmeditation war eine sich seit der Antike bis in die Moderne unter der intellektuellen Eliten bewährte Technik, die „als Perspektivenwechsel praktiziert [wurde], der dabei helfen sollte, sich aus der Blickrichtung eines »höheren Selbst«, eines "großen Anderen«, zu betrachten, der mit dem Allgemeinen - dem Gesetz der Polis, dem Kosmos, dem Göttlichen - verschmilzt. [...] Wer sich selbst als »tot« anschauen konnte, rettete sich auf die andere Seite der transzendentalen Differenz, wo der Doppelgänger zum Absoluten aufsteigt [...]. “12 Der Suizid wiederum steht der gesamten Menschheit als Selbsttechnik offen und wurde ebenfalls in vielen Fällen als Mittel zur Subjektspaltung eingesetzt:

9 Vgl. HADOT, Pierre (1991): Philosophie als Lebensform. Geistige Übungen in der Antike. Übersetzt von Ilsetraut Hadot und Christiane Marsch. Berlin; vgl. FOUCAULT, Michel (1993): Technologien des Selbst. In: LUTHER, Martin H. (Hg.): Technologien des Selbst. Übersetzt von Michael Bischoff. Frankfurt am Main.

10 MACHO, Thomas (2017): Das Leben nehmen. Suizid in der Moderne. Berlin; hier: S. 92.

11 Ebd, S. 93.

12 Ebd. 
„Seit dem Fin de Siècle, spätestens nach dem Ende des Zweiten Weltkriegs, hat sich die radikale Umwertung des Suizids - einerseits als Prozess der Enttabuisierung, andererseits als Verbreitung einer emanzipatorischen »Selbsttechnik« auf mehreren kulturellen Feldern vollzogen: als Protest in der Politik, als Strategie des Anschlags und Attentats in neueren Erscheinungsformen des bewaffneten Konflikts, als Grundthema der Philosophie und der Künste, in Literatur, Malerei und Film.“13

Inwiefern der Suizid auch für Elisabeth diese Funktion übernimmt, wird in der Textanalyse herausgearbeitet.

\section{2.}

Bereits das im Zuge der Aufklärung erstarkende Bürgertum sah im Konzept der Arbeit zusammen mit dem Leistungsdenken die konstituierenden Elemente, die als Trennlinie zum Adel begriffen wurden. Eine positive Konnotation von Arbeit war davor in der mitteleuropäischen Gesellschaft unbekannt. Bereits ein Blick auf die Wurzeln des deutschen Begriffs macht das klar: arbeit oder arebeit in mittelhochdeutschen Wörterbüchern mit „mühe, mühsal, not die man leidet od. freiwillig übernimmt“ übersetzt wurde. Erst mit der Entstehung des Protestantismus kommt auch eine positive Bewertung der Arbeit zustande: Arbeit wird nicht mehr als Leid angesehen, sondern als Ausformung der Nächstenliebe und Gottesfürchtigkeit. ${ }^{14}$ Jeder Gläubige zeigt seine Gottesfurcht durch ein werktätiges Leben, womit auch eine Kritik an die Nichtarbeitenden einhergeht - die sich vor allem gegen den Adel richtet. 1520 macht sich Martin Luther in seiner Schrift An den christlichen Adel deutscher Nation ${ }^{15}$ stark für diese Sichtweise und erhebt die Arbeit zum Werkzeug der Gotteshuldigung: „Ein Schuster, ein Schmied, ein Bauer, ein jeglicher hat seines Handwerks Amt und Werk, und dennoch sind sie alle gleich geweihte Priester und Bischöfe, und ein jeglicher soll mit seinem Amt oder Werk den anderen nützlich und dienstlich sein [...]." ${ }^{16}$ Diese und ähnliche Überlegungen werden zum Leitprinzip der mitteleuropäischen Gesellschaften bis zur Jahrhundertwende des 19. und 20. Jahrhunderts.

Seit diesen ersten gewichtigen positiven Bewertung des Arbeitsbegriffes sind gewaltige gesellschaftliche und technische Umwälzungen über Europa hinweggezogen: Die Erfindung der Dampfmaschine und damit auch der dampfbetriebenen Eisenbahn führten

13 Ebd, S. 8.

14 UNGER, Thomas (2013): Arbeit und Nichtarbeit in der Literatur. Texte dreier Jahrhundertwenden. In: BROGI, Susanna [et al.]: Repräsentationen von Arbeit. Transdisziplinäre Analysen und künstlerische Produktionen. Bielefeld, S. 59-86; hier: S. 62.

15 LUTHER, Martin: An den christlichen Adel deutscher Nation. Von des christlichen Standes Besserung (1520). Im heutigen Druck nach: Luther, Martin (1990): Ausgewählte Schriften. Band 1. Frankfurt am Main, S. 44-45.

16 Ebd., S. 17. 
zu einer exponentiellen Steigerungen der ökonomischen als auch sozialen Mobilität. ${ }^{17}$ Nicht nur die Mobilität beschleunigt sich, sondern auch die Kommunikationskanäle der Menschen. Telegraphen und Telefone ermöglichten - wenn auch in einem gesamtgesellschaftlich sehr eingeschränkten Maße - expandierende Erreichbarkeitsradien, die nicht nur im privaten Bereich Auswirkungen zeigten. Inmitten dieser Umbrüche erhält das Konzept Arbeit eine neue Deutung: Nicht mehr die Erfüllung von Tätigkeiten, sondern die fehlerlose Erfüllung von Tätigkeiten wird zu ihrem konstituierenden Bestandteil. Der amerikanische Ingenieur- und Arbeitswissenschaftler Frederick Winslow Taylor ${ }^{18}$ wurde zum Wegbereiter einer derartigen Denkweise. Er beobachtete menschliche Arbeitsabläufe und suchte nach Möglichkeiten der Vermeidung von unnötigen Krafteinsätzen während der einzelnen Handlungen. Durch seine wissenschaftliche Betriebsführung sollte das „Kraftsparsystem“ ${ }^{19}$ in jedem Unternehmen Einzug halten:

„Die Entwicklung einer wissenschaftlichen Methode bringt die Aufstellung einer Menge von Regeln, Gesetzen und Formeln mit sich, welche an Stelle des Gutdünkens des einzelnen Arbeiters treten. Sie können mit Erfolg erst angewendet werden, wenn sie systematisch aufgezeichnet und zusammengestellt sind. [...] Alle Kopfarbeit unter dem alten System wurde von dem Arbeiter mitgeleistet und war ein Resultat seiner persönlichen Erfahrung. Unter dem neuen System muß sie notwendigerweise von der Leitung getan werden in Übereinstimmung mit wissenschaftlich entwickelten Gesetzen. “20

Die Körper und Bewegungen der Arbeiter werden nicht mehr als Ganzes wahrgenommen, folglich verwundert auch nicht Taylors Degradierung des arbeitenden Individuums, wenn er schreibt: „Bisher stand die Persönlichkeit an erster Stelle, in Zukunft wird die Organisation und das System an erster Stelle treten“21. Die Überlegung Taylors fanden durch den Unternehmer Henry Ford Eingang in die Industriebetriebe, ${ }^{22}$ und manifestierten sich in der Perfektionierung der Fließbänder, die Ford zu der Feststellung brachte, dass diese nun „die Arbeit zu den Arbeitern hinschafften, statt umgekehrt“23. Ford setzte Taylors Forderungen konsequent durch: Zerlegung jeglicher Produktionsprozesse in kleinstmögliche Arbeitsschritte, eine geförderte Entlastung der Arbeiter von geistigen Tätigkeiten, sowie die Änderung des Lohnsystems sollen zu einer optimalen Nutzung der vorhandenen Leistungspotentiale führen. Das problematische Angleichen

17 Eine genaue Untersuchung zu den diversen Entwicklungen vgl. MALANIMA, Paolo (2010): Europäische Wirtschaftsgeschichte. 10.-19. Jahrhundert. Wien.

18 Frederick W. Taylor ist der Begründer des Scientific Management, das auch unter dem Namen Taylorismus bekannt wurde, aus der sich später die amerikanische Business administration entwickelt.

19 TAYLOR, Frederick W. (1977): Die Grundsätze wissenschaftlicher Betriebsführung. Hg. von Walter Volpert und Richard Vahrenkamp. Weinheim und Basel, hier: S. 29.

20 Ebd., S. 40.

21 Ebd., S. 4.

22 Vgl. UNGER [Anm. 14], S. 71.

23 FORD, Henry (1923): Mein Leben und Werk. Übersetzt von Curtt Thesing und Marguerite Thesing. Leipzig, hier: S. 93. 
der menschlichen und maschinellen Arbeitsabläufe wurde nicht nur negativ bewertet, da eine derartig beschleunigte Arbeitsleistung und Industrieanfertigung auch positive Effekte nach sich zogen: verringerte Warenpreise und höhere Arbeitslöhne. Thomas Unger weist auf die Rezeption von Fords Unternehmermodell im Deutschland der 1920er Jahre hin, so wurden in jenem Jahrzehnt mehr als 200000 Exemplare seiner Autobiographie Mein Leben und Werk (1923) verkauft. ${ }^{24}$ Die positiven (höhere Löhne, niedrigere Warenpreise) und negativen (immer gleiche Arbeitsschritte, verringerter Handlungsspielraum) Auswirkungen auf die Arbeitswelt fanden auch Ausdruck in der politischen Landschaft. Die Vertreterinnen und Vertreter der Arbeiterschaft reagierten uneins auf diese Entwicklung und formierten sich daher auch in unterschiedlichen Parteien:

\begin{abstract}
„Während kommunistische Gruppen den Fordismus ablehnten, weil er letztlich die Macht der Unternehmer zementierte, konnten Sozialdemokraten der Perspektive, Wohlstand für alle zu sichern, etwas abgewinnen. In diesem Zusammenhang ist daran zu erinnern, dass in der zweiten Hälfte des 19. Jahrhunderts im Zuge der Proletarisierung der Arbeitskräfte, die mit der Industrialisierung einherging, im Anschluss an Analysen und Forderungen von Karl Marx (1818-1883) und Friedrich Engels (1820-1895) eine breite Arbeiterbewegung entstanden war. Zur Zeit der Jahrhundertwende war sie als Sozialdemokratische Partei Deutschlands (SPD) eine Massenpartei und wichtige politische Kraft, die dann in der Weimarer Republik als Regierungspartei staatstragend werden sollte.“25
\end{abstract}

Die politischen Überlegungen der progressiven Kräfte fielen auf einen gesellschaftlichideengeschichtlichen Boden, der durch eine Schrift aus dem 19. Jahrhundert bereits ein bestimmtes Konzept von Arbeit und deren individuellen und sozialen Stellenwert kultiviert hatte: Wilhelm Heinrich Riehl ${ }^{26}$ (1823-1897) 1861 verfasste Schrift Die deutsche Arbeit. Für Riehl dient Arbeitsleistung der Legitimation und Erhöhung beziehungsweise Senkung der jeweiligen nationalen Volkspersönlichkeit. Die kollektive Volkspersönlichkeit steht dabei - ganz im Geiste des Sozialdarwinismus - in Konkurrenz zu anderen nationalen Volkspersönlichkeiten und führt auch einen Wettkampf ums Überleben: Denn „[f]aule Völker werden hinweggearbeitet von den fleißigeren, sie verschwinden auch ohne Gewaltt-

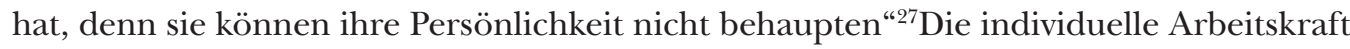
ist also auch der persönliche Beitrag zum nationalen Kollektiv, neben der individuellen Bedeutung erhält Arbeit mithin auch ein enormes Potential für einen gelingenden Ich-Entwurf, der sich in eine soziale Umwelt einfügen möchte und muss. Eine derartige

24 UNGER [Anm. 14], S. 71, Anm. 6.

25 UNGER [Anm. 14], S. 71.

26 Riehls Denken hat bis in die Gegenwart gewirkt. Durch seine Beobachtungen und Verarbeitung gesellschaftlicher Abläufe und Strukturen gilt er gemeinhin als eine der Gründungsfiguren der Soziologie. $\mathrm{Zu}$ biographischen Angaben sei auf folgenden Eintrag verwiesen: BRENDECKE, Arndt: Riehl, Wilhelm Heinrich von. In: Neue Deutsche Biographie (NDB). Band 21. Berlin 2003, S. 588-590, http://daten.digitalesammlungen.de/0001/bsb00016339/images/index.html?seite=602 (letzter Aufruf am 16.07.218). Zu Riehls frühsoziologischen Überlegungen und Ausformungen vgl. ALTENBOCKUM, Jasper von (1994): Wilhelm Heinrich Riehl 18231897. Sozialwissenschaft zwischen Kulturgeschichte und Ethnographie. Köln.

27 RIEHL, Wilhelm Heinrich (1861): Die deutsche Arbeit. Stuttgart, hier: S. 62. 
Haltung fordert auch Riehl, jeder und jede, die oder der Arbeit besitze müsse sich im Klaren sein, „daß er nicht bloß für sich und die Seinen, sondern zugleich auch für die Nation arbeitet“28 In den Jahren von 1860 bis 1928 erhöhte sich die Prestigeträchtigkeit der Arbeit und nahm im 20. Jahrhundert in den sich sukzessiv säkular entwickelnden Gesellschaften Europas gar die Position der Religion ein. ${ }^{29}$ Die Tücken dieser religiösen Aufladung zeigen sich allerdings, wenn einer derartig organisierten Gesellschaftsordnung die Arbeit ausgeht. Nach dem Ersten Weltkrieg und den goldenen 1920er Jahren der wirtschaftlichen Hochphase war es der Börsensturz Ende Oktober 1929 und in dessen Folge die Weltwirtschaftskrise, die Millionen von Menschen arbeitslos machte. Siegfried Kracauers 1930 erschienene soziologische Untersuchung Die Angestellten präsentierte exemplarische Fälle von Arbeitern und Angestellten diverser Berliner Großbetriebe, die lernen mussten mit der drohenden Arbeitslosigkeit umzugehen. ${ }^{30}$ Mit Blick auf die literarische Verarbeitung einer Angestellten-Existenz in Glaube, Liebe, Hoffnung ist Kracauers Beschreibung des gesellschaftlichen Klimas von Interesse:

„Über das Quantum der Sklaverei hier und dort läßt sich streiten, aber die Proletarisierung der Angestellten ist nicht zu bezweifeln. [...] Ferner ist die Existenzunsicherheit gewachsen und die Aussicht auf Unabhängigkeit nahezu völlig verschwunden.“31

Weiteren literarischen Ausdruck fand das soziale Elend dieser Zeit etwa bei Autoren wie Rudolf Brunngraber, Hans Fallada und Leonhard Frank im Arbeitslosenroman, ${ }^{32}$ aber auch im Film und Drama. Oft stehen kleine oder mittlere Angestellte, aber auch Industriearbeiter, und ihre unverschuldete Arbeitslosigkeit im Fokus der Arbeitslosenliteratur dieser Zeit. Wobei die Figuren dieser Texte ein subversives Potential besitzen:

\begin{abstract}
„Grundsätzlich aber zielen die Werke darauf ab, einer idealistischen Überhöhung von Arbeit entgegenzuwirken. Denn bei den arbeitslosen Romanfiguren ist keineswegs von einem Leben für die Arbeit die Rede, noch weniger von Arbeit als Religion, sondern es rücken ganz elementare Funktionen der Arbeit in den Vordergrund wie die Sicherung des Lebensunterhalts, eine sinnvolle Strukturierung des Alltags, ein anerkannter sozialer Status und Sozialkontakte außerhalb der familialen Primärbindungen. “33
\end{abstract}

Doch nicht nur anhand von Romanfiguren, sondern auch in Darstellungen von Arbeitslosen der damaligen Dramen werden diese Aspekte herausgestrichen.

28 Ebd., S. 108.

29 Vgl. HANK, Rainer (1995): Arbeit - die Religion des 20. Jahrhunderts. Auf dem Weg in die Gesellschaft der Selbständigen. Frankfurt am Main, hier: S. 10-12.

30 Vgl. KRACAUER, Siegfried (2013): Die Angestellten. Frankfurt am Main.

31 Ebd., S. 13.

32 Vgl. UNGER [Anm. 14].

33 Ebd., S. 77. 


\section{3.}

In den Randbemerkungen zum Drama Glaube, Liebe, Hoffnung gibt Ödön von Horváth Einblick in die Entstehungsgeschichte und Quellen des Dramas. Auf einer Durchreise im „Februar 1932“ (S. 9) in München ist Horváth auf einen Bekannten „namens Lukas Kristl“ (S. 9) getroffen, der als „Gerichtssaalberichterstatter“ (S. 9) tätig war und ihm von einem Fall berichtete hatte, der zur Grundlage des Dramas werden sollte:

„Und Kristl erzählte mir einen Fall aus seiner Praxis - und aus diesem alltäglichen Fall entstand der kleine Totentanz Glaube Liebe Hoffnung. Die Personen Elisabeth, den Schupo (Alfons Klostermeyer), die Frau Amtsgerichtsrat und den Oberinspektor hat Kristl persönlich gekannt. Es ist mir ein Bedürfnis, ihm auch an dieser Stelle für die Mitteilung seiner Materialkenntnisse und für manche Anregung zu danken." (S. 9)

Die Schilderung Kristls wurde auch in der sozialdemokratischen Münchner Post vom 13. bzw. 14. Juli 1929 veröffentlicht. Die Textarbeit selbst hat im Frühjahr 1932 begonnen, wobei Horváth auf Entwürfe rund um das Jahr 1930 zurückgegriffen zu haben scheint. Das durch eine gemeinsame Figurenkonstellation und -positionierung mit Kasimir und Karoline $^{34}$ eng verbundene Glaube, Liebe, Hoffnung schaffte erst nach der Bekanntschaft Horváths mit Kristl seinen Weg als eigenständiges Stück (vgl. 98-99), im Laufe der Vorarbeiten zeigte er sich auch für einige Szenenskizzen verantwortlich, wurde aufgrund einer Abmachung, aber nicht als Autor geführt:

„Ursprünglich wollten wir beide als Autoren zeichnen. Später meinte Horváth, die Berliner Kritiker nähmen ein Stück zweier Autoren nicht recht ernst. So kamen wir überein, nur auBerhalb Berlins gemeinsam zu zeichnen, bei der Berliner Premiere aber sollte er allein als Autor erscheinen und mich dafür im Programmheft erwähnen. Auf diese Weise entstand das Vorwort, das ich erst später zu Gesicht bekam.“ (S. 53)

Neben dem Wechsel innerhalb der Figurenbenennung, gab es auch Veränderungen im Titel. So waren In der Maschinerie der Paragraphen, In die Maschine geraten und Von der Maschine erfasst als mögliche Titel im Spiel. (S. 98) Vor dem Kontext einer vom Produktivitätsdenken Fords geprägten Wirtschaftsgebaren lesen sich diese Titelvorschläge wie kritische Anmerkungen jener Entwicklungen. Unter dem endgültigen Titel, Glaube, Liebe, Hoffnung, lag es schließlich im Sommer 1932 vor, und feierte im Januar 1933 seine Premier am Deutschen Theater Berlin. Unklar ist welche Fassung Horváth gespielt haben wollte, da es verschiedener Fassungen samt unterschiedlicher Untertitel gab: Kleinbürgerliche Komödie; Volksstück in zwei Teilen und einem Epilog; Volksstück in sieben Bildern; oder Ein kleiner Totentanz in fünf Bildern. (Vgl. 100) Horváth unternahm auch Einengungen beim Figurenpersonal und der Handlung vor - so fehlen etwa in späteren Fassungen Szenen zu einem Autounfall und Bierkeller. (vgl. 100) Das Stück sollte nach Geschichten

34 So hieß die Protagonistin ursprünglich in beiden Stücken Karoline; vgl. S. 99. 
aus dem Wiener Wald (1932) in einem Band unter dem Namen Glaube Liebe Hoffnung nebst Kasimir und Karoline. Zwei kleine Dramen aus dem Volksleben publiziert werden. Das Stück ging jedoch verloren und wurde erst 1961 im Band Stücke von Traugott Krischke herausgegeben. 1972 folgte dann durch die Gesammelten Werke ${ }^{35}$ die Veröffentlichung der Entwurfsvarianten und 1973 dann die Einzelausgabe ${ }^{36}$ samt Materialien. (S. 100)

\section{4.}

Zu Beginn seines Dramas evoziert Horváth das Bild einer naturwissenschaftlichmechanischen Gesellschaft:

„Erstes Bild

Szene Nummer 1

Schauplatz: Vor dem Anatomischen Institut mit Milchglasfenstern. Elisabeth will es betreten und sieht sich noch einmal fragend um, aber es ist nirgends eine Seele zu sehen. In der Ferne intoniert ein Orchester den beliebten Trauermarsch von Chopin und nun geht ein junger Schupo (Alfons Klostermeyer) langsam an Elisabeth vorbei und beachtet sie scheinbar kaum. Es ist Frühling. " (S. 13)

Der Ort des Geschehens ist das „Anatomische[] Institut“, die Einsicht in das Innere dieser wissenschaftlichen Institution ist von außen durch „Milchglasfenster[]“ gestört. Die Szene wird durch den musikalischen Hintergrund in Form von Chopins „beliebten Trauermarsch“, gemeint ist die Klaviersonate Nummer 2 oder auch die h-Moll, op. 58 genannt, ${ }^{37}$ ausgeschmückt, die neben der Todesassoziation auch eine Verbindung zur Abschlussszene herstellt. ${ }^{38}$

Die beiden zentralen Figuren Elisabeth und Alfons Klostermeyer werden vorgestellt und nach ihrem gesellschaftlichen Stand eingeordnet. Klostermeyer gehört durch seinen Beruf als Schutzpolizist zum Kleinbürgertum, Elisabeth ist als Arbeitslose von der gesellschaftlichen Ordnung ausgeschlossen. Die sich anbahnende Liebesbeziehung impliziert eine Standesproblematik:

„Szene Nummer 2

ELISABETH spricht den Schupo plötzlich an, während der Trauermarsch in der Ferne verhallt: Entschuldigens bitte - aber ich suche nämlich die Anatomie.

Schupo Das Anatomische Institut?

35 HORVÁTH, Ödön von (1972): Gesammelte Werke. 1. Volksstücke. Hg. Von Traugott Krischke. Frankfurt am Main.

36 HORVÁTH, Ödön von (1973): Glaube, Liebe, Hoffnung. Hg. Von Traugott Krischke. Frankfurt am Main.

37 Das Stück findet sich auch in Horváths Geschichten aus dem Wiener Wald; vgl. WÖHRLE, Dieter (2007): Kommentar. In: HORVÁTH, Ödön von (2007): Glaube, Liebe, Hoffnung. Ein kleiner Totentanz. Frankfurt am Main, S. 89-153, hier: S. 137.

38 Vgl. ebd. 
Elisabeth Dort wo man halt die Leichen zersägt.

Schupo Das dort ist das hier.

Elisabeth Dann ist es schon gut.

Stille.

SCHUPo lächelt: Gebens nur acht, Fräulein - da drinnen stehen die Köpf in Reih und Glied.

Elisabeth Ich habe keine Angst vor den Toten.

ScHUPO Ich auch nicht.“ (S. 13)

Neben des Kennenlernens der beiden Figuren wird in dieser Szene auch der Tod und der Umgang mit ihm von Elisabeth und Klostermeyer nicht nur angesprochen, ${ }^{39}$ sondern auch auf das Potential des Suizids als Selbsttechnik der Subjektspaltung verwiesen. Außer dem Polizeiapparat, in Gestalt des Schutzpolizisten Klostermeyer, tritt der Präparator als Vertreter des medizinisch-mechanischen Menschenbildes auf (vgl. S. 14). Die bis zur vierten Szene vorgestellten Figuren bilden Personifizierungen beinahe aller Gesellschaftsschichten ab: Elisabeth ([Lumpen]Proletariat), Alfons Kloster (Kleinbürgertum), Präparator (Bürgertum). Der fehlende Vertreter einer höheren Sozialschicht wird in der 5. Szene des ersten Bildes, präsentiert und dabei auch die Verflechtung der oberen Mittelschicht mit der adligen Oberschicht vorgeführt:

„Szene Nummer 5

Jetzt begleitet der Oberpräparator einen Baron mit Trauerflor aus dem Anatomischen Institut in das Freie.

OBERPRÄPARATOR Wird prompt erledigt, Herr Baron, und abermals mein innigstes Beileid.

BARON Danke, Herr Oberpräparator. Ich mache mir die heftigsten Vorwürfe.

OBERPRÄPARATOR Aber die staatsanwaltschaftlichen Erhebungen haben doch die völlige Haltlosigkeit der gegen Herrn Baron erhobenen etwaigen Beschuldigungen ergeben! Wir alle sind in Gottes Hand.

BARON Trotzdem ich stand vor Verdun und an der Somme, aber nichts hat mich so erschüttert, wie diese Katastrophe gestern. Wir waren ja erst seit drei Monaten verheiratet und ich steuerte den Unglückswagen - in der Unglückskurve. Zwischen Lechbruck und Steingaden. Nur gut, $\mathrm{da}$ der Leichnam freigegeben ist.

OBERPRÄPARATOR entdeckt inzwischen den Präparator: Augenblick bitte! Er nähert sich dicht dem Präparator und schreit ihn an. Sie füttern schon wieder die Tauben? [...].

PRÄPARATOR Aber das Fräulein dort wollte doch ihre Leiche verkaufen, Herr Oberpräparator -OBERPRÄPARATOR Ihre Leiche? Schon wieder?

Stille.

BARON Beispiellos.

OBERPRÄPARATOR Wir haben es zwar schon weißgottwieoft dementiert, daß wir keine solchen lebendigen Toten kaufen, aber die Leut glauben halt den amtlichen Verlautbarungen nichts! Die bilden sich gar ein, daß der Staat für ihren Corpus noch etwas daraufzahlen wird - gar so interessant kommen sie sich vor! Immer soll nur der Staat helfen, der Staat!

39 Ebd. 
BARON Eine völlig beispiellose Ansicht über die Pflichten des Staates.

OBERPRÄPARATOR Wird schon noch anders werden, Herr Baron.

BARON Hoffentlich." (S. 15-16)

Der Baron (Adel) und der Oberpräparator (Bürgertum) werden gesondert vorgestellt, und treten auch im Laufe des Dramas selten in Erscheinung, damit liegt der Fokus der Geschichte auf den unteren Gesellschaftsschichten. Im Gespräch zwischen dem Oberpräparator und Baron wird auch die Rolle des Staates und dessen Verhältnis zu seinen Bewohnerinnen und Bewohner angesprochen („Immer soll nur der Staat helfen, der Staat!").

Elisabeths wiederholte Anfrage, nun beim Vizepräparator, exemplifiziert die gesellschaftliche Notsituation, in der sie sich befindet und nimmt die sozialgeschichtlichen Gegebenheiten der Zeit und des Ortes der Entstehung auf. Damit ist die wirtschaftliche Rezession und Massenarbeitslosigkeit der beginnenden 1930er Jahre angesprochen, die auch durch den weiteren Verlauf des Dramas gezeichnet wird. Elisabeths Situation verbessert sich durch die Geldleihe des Präparators, die er vor seinem Vorgesetzten rechtfertigen muss:

„OBERPRÄPARATOR Zur Sache!

PRÄPARATOR Zur Sache: Ich werde dieser Zollinspektortochter unter die Arme greifen. Das steht bei mir felsenfest. Hundertfünfzig Mark.

OBERPRÄPARATOR Hundertfünfzig?

PrÄPARATOR Das Fräulein wird es mir schon zurückerstatten.“ (S. 21)

Die Freigiebigkeit des Präparators bei gleichzeitiger Benennung des Berufes ihres Vaters kommt nicht von ungefähr. Denn als staatliche Beamter wäre Elisabeths Vater kaum betroffen von der Entlassungswelle der 1930er Jahre und damit auch eine Rückerstattung seines Geldes gesichert. Das sozialkritische Element dieser Ausführungen, zeigt sich in der konkreten Nennung der Kosten von „[h]undertfünfzig Mark“, die den Lesern bemerkenswert erscheinen müssen, da in der ersten Szene des zweiten Bildes der Monatslohn des Amtsgerichtsrates mit „sechshundert Mark“ (S. 22) angegeben wird.

Das Drama etabliert bereits in den ersten Szenen alle sozialen Ebenen der Gesellschaft, gesellschaftliche Institutionen, als auch die Wechselwirkung zwischen Individuum und Staat, die durch die Rezession und Arbeitslosigkeit berührt werden. Außerdem wird die problematische Kopplung der Fremd- und Eigenwahrnehmung eines Individuums als Individuum („mir die Welt wieder öffnen“) innerhalb einer arbeitsteiligen Gesellschaft, die allein erwerbstätige Personen als Bestandteile der Gesellschaft anerkennt („fast selbstständige Position [...] in meiner ursprünglichen Branche“) in den Szenen des ersten Bildes aufgezeigt und in Elisabeths Begründung für die Geldleihe manifestiert:

„Elisabeth Sehens, wenn ich jetzt hundertfünfzig Mark hätt, dann könnt ich jetzt meinen Wandergewerbeschein haben und dann würde sich mir die Welt wieder öffnen - - weil ich mit einem Wandergewerbeschein schon morgen eine sozusagen fast selbständige Position bekom- 
men tät in meiner ursprünglichen Branche, aus der ich herausgerissen worden bin durch die Zeitumstände." (S. 18)

Das ausgeliehene Geld wird für Elisabeth zum Türöffner für die Arbeitswelt. Sie erhält erneut eine Anstellung im Damenbekleidungsgeschäft der Frau Prantl, die neben Elisabeth noch die Frau eines Amtgerichtsrates als Verkäuferin beschäftigt. Diese hat es aus ökonomischen Gründen nicht nötig zu arbeiten, möchte jedoch ihren Platz in der sozialen Oberschicht trotz Rezession weiter ausbauen - allerdings unter Vorspiegelung falscher Tatsachen:

Frau Amtsgerichtsrat Aber es bleibt doch dabei: Wenn jemand fragen sollte, dann sagen Sie selbstredend, ich verkaufe das nur von wegen persönlicher Zerstreuung und so

Die Prantl Ist doch sonnenklar, wollte sagen: bleibt unter uns!

Frau AmtsgerichtsRat Bei diesen schweren Zeiten muß man auch dem eigenen Manne unter die Arme greifen, der verdient jetzt noch ganze sechshundert Mark. Da wird abgebaut und abgebaut, aber die Herren Landgerichtsdirektoren und Ministerialräte - Sie stockt, da das Telefon klingelt. "(S. 22-23)

Die Institution Erwerbsarbeit und deren Präfigurierung für die gesellschaftliche Rolle des Individuums wird durch das Aufzeigen der unterschiedlichen Bewertungshorizonte angezeigt. Die Frau Amtsgerichtsrat hätte keine zusätzlichen Einkünfte notwendig, allerdings nimmt sie die zusätzliche Einnahmequelle in Anspruch, auch vor dem Hintergrund einer zukünftigen Verschlechterung der wirtschaftlichen Lage („Da wird abgebaut und abgebaut“). Für Elisabeth ist der Fall anders gelagert. Da sie das Geld nicht nur für Ihren Wandergewerbeschein, sondern auch die Bezahlung einer früheren Geldstrafe benötigt, ist für sie das Angestelltenverhältnis nicht nur eine Möglichkeit zu arbeiten, sondern auch mit dem Zugang zu gesellschaftlicher Beachtung verbunden. Die Wahrnehmung des einzelnen Menschen durch die Gesellschaft wird im Drama an die Ausübung eines Berufes gekoppelt - anders ausgedrückt: Wer als jemand beachtenswertes wahrgenommen werden will, muss als Vorbedingung dafür eine Arbeitsstelle nachweisen. Diese gesellschaftlichen Umstände sind es, die Elisabeth in eine unangenehme Situation bringen. In Prantls Bekleidungsgeschäft offenbart sie, dass sie sich 150 Mark vom Präparator geliehen hatte, diese allerdings nicht für den ersehnten Wandergewerbeschein, sondern für die Bezahlung einer in der Vergangenheit liegenden Geldstrafe benötigt habe. Gleichzeitig stellt sich heraus, dass sie - anders als von ihr in der ersten Szene erklärt - bereits einen Wandergewerbeschein von Prantl erhalten hatte.

Nach Elisabeths Kündigung durch Prantl kommt es noch zu einem kurzen Gespräch zwischen ihr und der Frau Amtsgerichtsrat:

„Frau Amtsgerichtsrat Mich geht es ja nichts an, aber vorbestraft ist immer schon arg.

ELISABETH sagt es auf wie ein Schulmädchen: Ich bin vorbestraft, weil ich ohne Wandergewerbeschein gearbeitet habe - und da hat man mir eine Geldstrafe von einhundertundfünfzig Mark 
hinaufgehaut, bezahlbar in Raten. Aber dann ist alles fällig geworden und ich hätt dafür in das Gefängnis müssen und meine Zukunft wäre wieder in das Wasser gefallen - und so habe ich dafür dem Herrn Präparator sein Geld aufgebraucht.“

Elisabeths Erklärung verdeutlicht die schiefe Lage zwischen ihr und den staatlichen Institutionen. Denn nachdem ihr eine Ratenzahlung der Strafe gewährt worden war („bezahlbar in Raten“), ist es zu einer plötzlichen Aufforderung zur Zahlung der Gesamtsumme gekommen („dann ist alles fällig geworden“). In ihrer Schilderung verzichtet Elisabeth auf jegliche Euphemismen, wie Wöhrle bemerkt, entspricht das Horváths Ansicht, dass sich Menschen bloß in den seltensten Fällen als Menschen zeigen. ${ }^{40}$

In den bisher gezeigten Szenen wird immer wieder das Verhältnis eines Individuums zum Staat gezeigt, dabei ist auch an den „Schlüsselsatz“ der Randbemerkungen zu denken, der erklärt, es bestehe ein „gigantischer Kampf zwischen Individuum und Gesellschaft“ (S. 10). In der ersten ausführlichen Deutung des Stückes Ödön von Horváth als Kritiker seiner Zeit kommt Axel Fritz 1973 auf ein nachvollziehbares Fazit:

„wie die moralischen Vorurteile einer bürgerlich-mittelständisch ausgerichteten Gesellschaft den einmal Vorbestraften - und seien Delikt und Strafe noch so geringfügig - nicht mehr als vollwertiges Mitglied ihrer Gesellschaft akzeptieren, sondern ihn durch ihr Verhalten geradezu zwingen, sich durch neue Verstöße über Wasser zu halten [...].“41

Fritz‘ Diagnose ist zuzustimmen, es sind die „die moralischen Vorurteile einer bürgerlich-mittelständisch ausgerichteten Gesellschaft“, die Elisabeth die soziale Zugehörigkeit entziehen und sie zu neuen Normüberschreitung und in weiterer Folge zu einer Verletzung der Moralvorstellungen der Gesellschaft bringen. So geschieht es, dass Elisabeth nach ihrer Kündigung beim Wohlfahrtsamt mit dem Schutzpolizisten Klostermeyer zusammenkommt. Die Beiden werden zu Beginn des vierten Bildes in Elisabeths „möblierte[n] Zimmer“ (S. 44) durch einen „Oberinspektor der Sittenpolizei“ (S. 47) überrascht.

Hier manifestiert sich auch die von Ingrid Haag 1995 im Artikel Ödön von Horváth. Fassaden-Dramaturgie. Beschreibung einer theatralischen Form aufgestellte These, dass Elisabeth im gesamten Dramenverlauf von staatlichen Verwaltungsinstanzen abgewiesen werde und dies sich im strukturgebenden „Spiel von Zeigen und Verbergen“42 zeige: Abweisung durch das Anatomische Institut oder die Nichtzuständigkeit durch das Wohlfahrtsamt. ${ }^{43}$ Und auch in der Szene mit Klostermeyer und dem Oberinspektor zeigt sich eine gesellschaftliche Abweisung. Denn statt Schutz und Zuflucht bei einem Organ der Exekutive zu erhalten, erwartet Elisabeth in der Gesellschaft der Polizisten eine weitere Demütigung.

40 Ebd., S. 143.

41 FRITZ, Axel (1973): Ödön von Horváth als Kritiker seiner Zeit. München, hier: S. 154.

42 HAAG, Ingrid (1995): Ödön von Horváth. Fassaden-Dramaturgie. Beschreibung einer theatralischen Form. Frankfurt am Main, hier: S. 7.

43 Ebd., S. 84. 
Ein arbeitsloses Individuum scheint für die staatlichen Institutionen, aber auch für große Teile der Gesellschaft der weiteren Auseinandersetzung nicht wert - man denke nur an das abweisende Verhalten der diversen Figuren, die ebenso wie auf Elisabeths Bestrafung auch auf Ihre Arbeitslosigkeit mit Abweisung reagieren. Der Suizid ermöglicht Elisabeth eine Subjektspaltung, innerhalb derer es ihr ermöglicht wird Eintritt in die Institutionen zu erhalten:

„Die Tatsache, daß sich für Elisabeth, die ewig abgewiesene Bittstellerin, zum ersten und letzten Mal die Tür einer Verwaltungsinstanz öffnet, schreibt sich ein in eine Serie zynischer Umkehrungen: Die Lebenden, die an der >Nichtzuständigkeit $>$ der Ämter verzweifelte, wird als Sterbende saufgenommen< [...]. “44

Der Weg zur Sterbenden und in Elisabeths Fall zur Suizidantin ist in einer fehlerhaften Beziehung zwischen der Arbeitslosen und der Gesellschaft - sowohl auf Makro- als auch Mikroebene - zu sehen, auch hier kommt das Prinzip des Verbergens der Verursacher zum Einsatz, und eine genaue Lektüre zeigt auf, dass

„die Gerichtsinstanz selbst, die Elisabeths `Todesurteil< ausspricht, nicht gezeigt wird. Die Unsichtbarkeit der Instanz bedeutet deren Allgegenwart. Jede Station auf Elisabeths Weg, wird zum Tribunal, wo hinter der Fassade privat-intimer, freundschaftlich-väterlicher Rede Elisabeths Verurteilung vorbereitet, ausgesprochen, wiederholt und bestätigt wird.“45

Elisabeth ist mit ihrem Versuch, durch Arbeit ein gelingendes Subjektbewusstsein zu entwickeln, gescheitert - ihre Interaktionsversuche haben ihre soziale Position sogar noch verschlechtert. In den Szenen des vierten Bildes wird diese Problematik im Gespräch mit Klostermeyer sichtbar. Elisabeth ist es durch ihre Liebesbeziehung mit dem Polizisten gelungen sich sukzessiv als subjektives Ich einer Gemeinschaft zu verstehen. Das unangekündigte Eindringen des Oberinspektors in ihr Zimmer bricht diesen beinahe abgeschlossenen Prozess abrupt ab:

„Elisabeth Was will man denn von mir?

Oberinspektor Sie haben von der Polizei einen Unterkommensauftrag gekriegt, darauf steht, daß Sie sich innerhalb dreier Wochen um ein einwandfreies Unterkommen umsehen sollen. Aber Sie haben weder Arbeit noch haben Sie nachgewiesen, daß Sie sich um eine solche bemüht haben.

ELISABETH Kümmern Sie sich doch um die Leut, die kein Unterkommen haben!

OberinsPektor Keine Hetzreden bitte! Polizeiwidrig ist nicht, wer kein Unterkommen hat, polizeiwidrig ist nur, wer dadurch die öffentliche Ordnung gefährdet.

ELISABETH Aber ich gefährde doch nicht die öffentliche Ordnung!

44 Ebd., S. 87.

45 Ebd., S. 91. 
ObERINSPEKTOR Solange Sie sich nicht über Ihre Einkünfte ausweisen können, ist dies fraglich.

Elisabeth Für mich wird schon gesorgt.“ (S. 48)

Der Verweis auf die fehlende Unterkunft Elisabeths wird als Vorwurf formuliert und gleichzeitig mit einem staatlichen Instrument („Unterkommensauftrag“) verbunden. Der folgende Satz ist nun von enormer Bedeutung für die hier vorgestellte Lesart: „Aber Sie haben weder Arbeit noch haben Sie nachgewiesen, daß Sie sich um eine solche bemüht haben." Nicht nur tadelt der Oberinspektor die fehlende Arbeitsstelle, sondern auch die - von ihm angenommene - fehlende Initiative, „sich um eine solche bemüht [zu] haben." Im folgenden Schritt verlässt er den an ein Subjekt gerichteten Vorwurf und nimmt eine Abstraktion vor, innerhalb derer Elisabeth als Gefahr für die Öffentlichkeit gebrandmarkt wird: „Polizeiwidrig ist nicht, wer kein Unterkommen hat, polizeiwidrig ist nur, wer dadurch die öffentliche Ordnung gefährdet.“ Auf die Frage nach ihrem Einkommensnachweis reagiert Elisabeth mit einer sachlichen Replik („Für mich wird schon gesorgt“). Die Nachfrage des Oberinspektors nach „diese[r] freundliche[n] Fürsorge“ (S. 48) zeigt die Tücke in Elisabeths derzeitiger sozialer Bindung zu Klostermeyer: „Ich erhalte von meinem Bräutigam zwanzig Mark in der Woche. Davon lebe ich." (S. 48) Die Beziehung zu Klostermeyer zeichnet sich nicht nur durch emotionale Zuneigung aus, sondern durch ökonomische Abhängigkeit. Nur zwei Szenen weiter zeigt sich die Brisanz einer solchen Beziehung innerhalb eines gesellschaftlichen Klimas, das Arbeit als essentielles Identitätsmerkmal ansieht. So wird Klostermeyer in der 10 Szene des vierten Bildes Elisabeths kriminelle Vergangenheit mitgeteilt - mit dem Hintergedanken den Schutzpolizisten von einer Ehe abzubringen:

„OBERINSPEKTor Bedaure, aber Sie scheinen es nicht zu ahnen, wen Sie da an den Traualtar führen wollen - - Ihre Braut hat doch wegen Betrug bereits vierzehn Tage Gefängnis hinter sich. SCHUPO Gefängnis?

Oberinspektor Betrug. Abgesehen von einer Geldstrafe, die sie sich auch schon mal geholt hat. Daß diesen Damen derlei Verbindungen mit der Polizei ganz erwünscht sind, ist ja menschlich verständlich. Aber ob das Ihrer Karriere sehr förderlich ist - -

Schupo Keine Ahnung - -“ (S. 50)

In der folgenden Szene 11 spricht Elisabeth an, was Klostermeyer zu schaffen macht. Es sei nicht ihre Unaufrichtigkeit, die ihn zum Gehen veranlasst habe, sondern es seien die drohenden Folgen für seine Karriere:

„SCHUPO Du hast mich belogen und das ist für mich der entscheidende Punkt.

Elisabeth Nein, deine Karriere, das ist er, dein entscheidender Punkt.

Schupo Nein! Aber zuerst kommt die Pflicht und dann kommt noch Ewigkeiten nichts! Radikal nichts!

Stille“ (S. 51)

Mit dem Verweis auf seine (Arbeits)Pflicht, bekräftigt Klostermeyer den von ihm bereits an früherer Stelle geäußerten - „Pflicht ist Pflicht“ (S. 42) - Stellenwert seiner Erwerbsarbeit 
für seine eigene Identität („dann kommt noch Ewigkeiten nichts!“). Nachdem Klostermeyer das Zimmer verlässt, kommt es zu einem kurzen Gespräch zwischen Elisabeth und dem Oberinspektor. Elisabeth ist keineswegs bloßes Opfer, sondern sieht mit dem Verlust Klostermeyers auch die Chance auf ein erneutes Eintreten in die Arbeitswelt wegbrechen:

\author{
„OBERINSPEKTOR Also das war wirklich nicht notwendig von Ihnen, dem Mann seine Karriere \\ so leichtfertig zu gefährden - \\ ELISABETH Notwendig? Und meine Karriere? \\ OBERINSPEKTOR Sie wollen doch nicht behaupten, daß Sie unschuldig sind? \\ EuISABETH $O$ nein, das habe ich mir schon längst abgewöhnt. Entschuldigens, aber jetzt muß \\ ich lachen - - \\ Sie setzt sich auf den Bettrand und lacht lautlos. \\ OBERINSPEKTOR Lachens Ihnen nur ruhig aus.“ (S. 52)
}

Der letzte Satz des Oberinspektors irritiert, was auch Dieter Wöhrle 2007 in seiner Beobachtung Ausblick: Warum bei Horváth das einzelne Wort zählt bemerkt, „»Weinen sie sich ruhig aus!« dürfte dementsprechend die situationsgerechte Aufforderung lauten" (S. 124). Bleibt man bei der gedruckten Version, und behält die von Wöhrle vorgeschlagene Variante als implizite Konnotation im Hinterkopf, zeigt sich die Aussage als imperative Diagnose: Elisabeth ist durch das Scheitern ihrer Beziehung zu Klostermeyer auch die letzte Möglichkeit einer gesellschaftlichen Inklusion - bei gleichzeitiger Exklusion durch Identifizierung mit einer Erwerbsarbeit - abhanden gekommen, das sich Auslachen wird zur symbolischen Handlung einer gestörten Verbindung zwischen einem Individuum und seiner Umwelt.

Nachdem Elisabeth nach ihrem Suizidversuch in das Polizeirevier geführt wird und nach einigen Momenten bei vollem Bewusstsein zu sein scheint, äußert sie ihr Misstrauen und ihr Widerstand gegen eine erneute Inbetriebnahme eines Inklusions- und Exklusionsentwurfs ihrer Identität innerhalb einer jegliche Kommunikation blockierenden sozialen Umwelt: „Elisabeth Jetzt war ich schon fort und jetzt gehts wieder los und niemand ist zuständig für dich und du hast so gar keinen Sinn -" Auch Wöhler bemerkt Elisabeths gewandelte Beschreibung ihrer Situation, „[d]abei fällt sie aus der Ich-Perspektive und der Rolle als Subjekt, indem sie plötzlich mit sich selbst spricht, sich als Person mit dich/du anspricht und sich damit zum Objekt macht.“ (S. 121) Elisabeth gibt jeglichen Versuch „als selbst bestimmendes Subjekt" zu agieren auf, und präsentiert sich selbst „als Objekt“ (S. 122). In der 14. Szene zeigen sich noch stärkere Züge einer Subjektspaltung:

„ElisABETH Ich rede jetzt nicht direkt persönlich, denn ich bin darüber momentan hinaus - Sie brüllt ihren Alfons plötzlich an. Glotz mich nicht so an! Geh mir aus den Augen, sonst reiß ich mir die Augen aus! Bild dir doch nicht ein, daß ich wegen dir ins Wasser bin, du mit deiner großen Zukunft! Ich bin doch nur ins Wasser, weil ich nichts mehr zu Fressen hab - wenn ich was zum Fressen gehabt hätt, meinst, ich hätt dich auch nur angespuckt? Schau mich nicht so an!! Sie wirft mit der Schnapsflasche nach seinen Augen, verfehlt aber ihr Ziel. Da!“ (S. 63-64) 
Nicht nur, dass jegliches persönliche Sprechen („nicht direkt persönlich“) entfällt, sondern auch Elisabeths Verweigerung der Fremdwahrnehmung als Subjekt („Schau mich nicht so an!!!“) unterstreichen diesen Prozess. Und auch der Wandel weg vom menschlichen hin zum animalischen Verhalten belegt die Folgen - der missglückten Subjektspaltung, sei es etwa durch irrational-aggressive Handlungen:

„VIzePräPARATOR Was? Beißen wirst du - beißen?!

ELISABETH zieht sich verschüchtert zurück.

BuCHHALTER Jetzt beißt sie ihren eigenen Lebensretter -

ELISABETH fletscht die Zähne." (S. 64)

Aber auch die Interaktion mit den anderen Figuren macht die Auswirkungen deutlich:

„VIZEPRÄPARATOR Was knurrt denn da?

BuchHalter Dem Fräulein ihr Magen.

Dritter Schupo zum Kameraden: Hast nichts dabei?

KAMERAD Doch - Er gibt aus seiner Manteltasche Elisabeth ein Brötchen.

ELISABETH nimmt es apathisch und knabbert daran.

DRITTER SCHUPO zieht sich ebenfalls seine weißen Handschuhe an: Schmeckts?

ELISABETH lächelt apathisch - plötzlich läßt sie das Brötchen fallen und sinkt über den Tisch." (S. 64-65)

Erst unmittelbar vor ihrem Ableben zeigt Elisabeth wieder Anzeichen eines menschlichen Subjekts, mit der Aussage, sie „lasse den Kopf nicht hängen“ (S. 65) blitzt gar Optimismus auf, der jedoch nicht mehr fruchten kann: "Sie schlägt mit der Hand in die Luft, als würde sie Fliegen abwehren. Na! Da fliegen lauter so schwarze Würmer herum Sie stirbt sanft." (S. 65)

Am Ende bietet sich der arbeitslosen Elisabeth keine Möglichkeit an ihre Umwelt anzuknüpfen und sich auf Basis ebendieser zu sich selbst zu beziehen, zu exkludieren, um dadurch die durch die arbeitsteilige Gesellschaft latent fragmentierten Subjekt-Bestandteile einem zumindest situativ stabilen Ich-Entwurf zu inkludieren. Als Notlösung vollzieht sie eine Subjektspaltung - die sich als tranceartiger Zustand präsentiert. Die problematischen sozioökonomischen Mechanismen beschreibt Wöhrle treffend mit dem Bild eines Teufelskreises, der nicht zu unterbrechen sei, „solange gilt, dass Elisabeth Geld benötigt, um arbeiten zu können, und sie Arbeit braucht, um Geld zu verdienen, solange gelten auch Glaube, Liebe und Hoffnung als die zwar geforderten, aber erfolglosen Tugenden." (S. 125)

Nachdem Elisabeth eine Selbstkonstituierung innerhalb der reduzierten interpersonellen als auch ökonomischen Möglichkeiten einer absoluten Individualitätserfahrung verwehrt wird, ihre Anknüpfungsversuche zu verschiedenen Bezugssystemen - konkret Erwerbsarbeit und Liebe - scheitern, nimmt sie schließlich eine Spaltung ihres fragmentarischen Subjektbewusstseins vor. Die Handlung ist symbolisch aufgeladen, und lässt an Immanuel Kants Selbstzweckformel denken, der konstatiert, dass ein Mensch, der, 
„um einem beschwerlichen Zustande zu entfliehen, sich selbst zerstört, [...] sich einer Person bloß als eines Mittels zur Erhaltung eines erträglichen Zustandes bis zu Ende des Lebens“46 bediene. Der oder die Selbstmörderin verwende das eigene Leben als Mittel zum Erreichen einer wie auch immer gearteten Erlösung - in Elisabeths Fall vom gescheiterten Ich-Entwurf - und nicht als Selbstzweck. ${ }^{47}$ Anders gesagt: der Suizid ist die absolute Objektivierung des Menschen. Ebendies wurde in diesem Stück exemplarisch vorgeführt und reiht sich damit in eine poetologische Verfahrensweise Horváths, die sich in seinem Gesamtwerk erkennen lässt.

\section{Literaturverzechnis}

ALTENBOCKUM, Jasper von (1994): Wilhelm Heinrich Riehl 18231897. Sozialwissenschaft zwischen Kulturgeschichte und Ethnographie. Köln.

BLOCHER, Friedrich K. (1984): Identitätserfahrung. Literarische Beiträge von Goethe bis zu Walser. Köln.

BRENDECKE, Arndt: Riehl, Wilhelm Heinrich von. In: Neue Deutsche Biographie (NDB). Band 21. Berlin 2003, S. 588-590, http://daten.digitalesammlungen.de/0001/bsb00016339/ images/index.html?seite=602 (letzter Aufruf am 16.07.2018).

FORD, Henry (1923): Mein Leben und Werk. Übersetzt von Curtt Thesing und Marguerite Thesing. Leipzig.

FOUCAULT, Michel (1993): Technologien des Selbst. In: Luther, Martin H. (Hg.): Technologien des Selbst. Übersetzt von Michael Bischoff. Frankfurt am Main.

FRITZ, Axel (1973): Ödön von Horváth als Kritiker seiner Zeit. München.

HAAG, Ingrid (1995): Ödön von Horváth. Fassaden-Dramaturgie. Beschreibung einer theatralischen Form. Frankfurt am Main.

HADOT, Pierre (1991): Philosophie als Lebensform. Geistige Übungen in der Antike. Übersetzt von Ilsetraut Hadot und Christiane Marsch. Berlin.

HANK, Rainer (1995): Arbeit - die Religion des 20. Jahrhunderts. Auf dem Weg in die Gesellschaft der Selbständigen. Frankfurt am Main.

HORVÁTH, Ödön von (2007): Glaube, Liebe, Hoffnung. Ein kleiner Totentanz. Frankfurt am Main.

HORVÁTH, Ödön von (1972): Gesammelte Werke. 1. Volksstücke. Hg. von Traugott Krischke. Frankfurt am Main.

HORVÁTH, Ödön von (1973): Glaube, Liebe, Hoffnung. Hg. von Traugott Krischke. Frankfurt am Main.

KRACAUER, Siegfried (2013): Die Angestellten. Frankfurt am Main.

KANT, Immanuel (2004): Grundlegung zur Metaphysik der Sitten. Stuttgart.

LUHMANN, Niklas (1998): Individuum, Individualität, Individualismus. In: Luhmann, Niklas: Gesellschaftsstruktur und Semantik. Studien zur Wissenssoziologie der modernen Gesellschaft. Bd. 3. (2. Aufl.). Frankfurt am Main, S. 149-258.

LUTHER, Martin (1990): Ausgewählte Schriften. Band 1. Frankfurt am Main.

MACHO, Thomas (2017): Das Leben nehmen. Suizid in der Moderne. Berlin.

46 KANT, Immanuel (2004): Grundlegung zur Metaphysik der Sitten. Stuttgart, hier: S. 79-80.

47 Ebd. 
MALANIMA, Paolo (2010): Europäische Wirtschaftsgeschichte.10.-9. Jahrhundert. Wien.

RIEHL, Wilhelm Heinrich (1861): Die deutsche Arbeit. Stuttgart.

TAYLOR, Frederick W. (1977): Die Grundsätze wissenschaftlicher Betriebsführung. Hg. von Walter Volpert und Richard Vahrenkamp. Weinheim und Basel.

UNGER, Thomas (2013): Arbeit und Nichtarbeit in der Literatur. Texte dreier Jahrhundertwenden. In: Brogi, Susanna [et al.]: Repräsentationen von Arbeit. Transdisziplinäre Analysen und künstlerische Produktionen. Bielefeld, S. 59-86.

WÖHRLE, Dieter (2007): Kommentar. In: Horváth, Ödön von (2007): Glaube, Liebe, Hoffnung. Ein kleiner Totentanz. Frankfurt am Main, S. 89-153.

Erkan Osmanović, MA / osmanovic@phil.muni.cz

Masarykova univerzita, Filozofická fakulta, Ústav germanistiky, nordistiky a nederlandistiky Arna Nováka 1, 60200 Brno, CZ 